\title{
Utilizing a Professionalism Mentor to Address Sexual Harassment in Academic Ophthalmology
}

Michelle T. Cabrera, MD ${ }^{1,2}$ Karen L. Christopher, MD 3 Megan E. Collins, MD ${ }^{4}$ Eliesa Ing, MD

Grace Sun, $\mathrm{MD}^{6}$ Jeff H. Pettey, MD Shira S. Simon, $\mathrm{MD}^{8}$ Jayanth Sridhar, MD Jeffrey R. Soohoo, MD ${ }^{3}$ Rachel G. Simpson, $M^{7} \quad$ Leona Ding, $M^{1} \quad$ Stacy L. Pineles, MD ${ }^{10}$

${ }^{1}$ Department of Ophthalmology, University of Washington, Seattle, Washington

2 Department of Ophthalmology, Seattle Children's Hospital, Seattle, Washington

${ }^{3}$ Department of Ophthalmology, University of Colorado School of Medicine, Aurora, Colorado

${ }^{4}$ Wilmer Eye Institute, Johns Hopkins University School of Medicine, Baltimore, Maryland

${ }^{5}$ Casey Eye Institute, Oregon Health \& Science University, Portland, Oregon

${ }^{6}$ Department of Ophthalmology, Weill-Cornell Medicine,

New York City, New York

${ }^{7}$ Department of Ophthalmology and Visual Sciences, John A. Moran Eye Center, Salt Lake City, Utah

8 Department of Ophthalmology, Northwestern University, Evanston, Illinois

${ }^{9}$ Department of Ophthalmology, Bascom Palmer Eye Institute, Miami, Florida

${ }^{10}$ Department of Ophthalmology, Stein Eye Institute, UCLA, Los Angeles, California

J Acad Ophthalmol 2021;13:e11-e18
Address for correspondence Michelle T. Cabrera, MD, OA.9.220; 4800 Sand Point Way NE, Seattle, WA 98105

(e-mail: michelle.cabrera@seattlechildrens.org).

\author{
Abstract \\ Keywords \\ - sexual harassment \\ - faculty \\ - ophthalmology \\ - graduate medical \\ education \\ - residency \\ - academic medicine \\ - professionalism \\ mentor \\ - education \\ - Title IX \\ - prevention
}

Objective This study assesses a new departmental role-a professionalism mentorwho receives sexual harassment reporting, liaisons with campus resources, and organizes educational sessions.

Study Design Multicenter randomized controlled survey study. Methods Academic ophthalmology departments in the United States were randomized to a professionalism mentor group $(n=9)$ and a control group $(n=7)$. Among both pre- and postsurveys, 605 faculty and trainee responses were received and 546 were complete. The intervention group was assigned a professionalism mentor with educational session for a 6 - to 10 -month period. Sexual harassment and reporting rate change over time were compared between the two groups.

Results Among 546 anonymous responses, 16\% experienced workplace sexual harassment during the prior 10 months. Location in the South or Midwest was a risk factor $(p<0.001)$. Victims were mostly women $(76 \%)$, including residents/fellows $(46 \%)$ and academic attendings (49\%); perpetrators included patients $(35 \%)$ and academic attendings (35\%). Departments with and without a professionalism mentor received

September 12, 2020 accepted after revision November 18, 2020
DOI https://doi.org/ 10.1055/s-0040-1722745. ISSN 2475-4757.
(C) 2021. The Author(s).

This is an open access article published by Thieme under the terms of the Creative Commons Attribution-NonDerivative-NonCommercial-License, permitting copying and reproduction so long as the original work is given appropriate credit. Contents may not be used for commercial purposes, or adapted, remixed, transformed or built upon. (https://creativecommons.org/ licenses/by-nc-nd/4.0/)

Thieme Medical Publishers, Inc., 333 Seventh Avenue, 18th Floor, New York, NY 10001, USA 
had stable harassment from pre- to postsurvey ( $p=0.95$ comparing change). The professionalism mentor group had an increase in reporting to an authority from pre- to postsurvey (7-23\%), whereas the control group had a decrease $(27-12 \% ; p=0.07$ comparing change). Most faculty and trainees in the interventional arm of this study recommended instituting a professionalism mentor with educational session $(66 \%$ presurvey and $68 \%$ postsurvey), compared with educational session alone (25\% presurvey and $23 \%$ postsurvey), or neither ( $9 \%$ presurvey and $9 \%$ postsurvey). Residency program directors in the professionalism mentor group even more strongly supported instituting both a professionalism mentor and educational program (100\% presurvey and $100 \%$ postsurvey) as opposed to educational program alone ( $0 \%$ presurvey and $0 \%$ postsurvey) or neither ( $0 \%$ presurvey and $0 \%$ postsurvey).

Conclusion This study identified a high sexual harassment rate in academic ophthalmology departments over a brief period. The presence of a professionalism mentor was viewed favorably and may lead to increased reporting.

In a recently published anonymous survey study, ${ }^{1} 59 \%$ of female ophthalmologists reported experiencing sexual harassment in the workplace, with the majority of experiences occurring during residency training. These results are consistent with other studies regarding sexual harassment in academic medicine (range $=30-59.4 \%),{ }^{2-4}$ and surgical fields in particular have reported higher rates of sexual harassment (60\%). ${ }^{5}$ In the ophthalmology study, ${ }^{1}$ only $15 \%$ of victims reported their most significant experience to an authority. Particularly troubling were described cases of coercion by department chairs and residency program directors. ${ }^{1}$ Federal Title IX policy requires that universities provide resources to report and investigate sexual harassment or assault; however, these resources are often unfamiliar, and victims of assault may not feel comfortable reporting such sensitive incidents. Furthermore, faculty and trainees may not feel that their department supports reporting through such pathways and may fear retaliation. $^{6}$

We are proposing a new departmental role, called a professionalism mentor (an approachable internal faculty member(s) independent from departmental leadership who coordinates education regarding sexual harassment and gender discrimination), who receives reporting and could serve as liaison with the university's Title IX department. By providing such a role in addition to existing resources and reporting pathways, faculty and trainees would have an alternate local departmental structure for education and reporting outside of residency program directors and chairs, who may be intimidating or may themselves be the perpetrators. Furthermore, departmental structure that includes a professionalism mentor sends a strong message to faculty and trainees that the department takes the issue of sexual harassment seriously and supports reporting and prevention.

This randomized controlled study assigned academic ophthalmology departments to a professionalism mentor group and a control group for a 6- to 10 -month period, anonymously assessing the opinions and experiences of residents, fellows, and faculty both before and after the study period. The goal of this study was to identify an impact on rate and reporting of sexual harassment, with a secondary goal to understand satisfaction of participants in this program. This study also collected opinions from participating residency program directors and professionalism mentors.

\section{Methods}

This prospective and multicenter study received institutional review board (IRB) approval at the University of Washington for administration of online anonymous surveys to faculty and trainees at 16 participating academic ophthalmology departments. The IRB approval or exemption was obtained at all participating study sites, and this work adhered to the tenets of the Declaration of Helsinki and the Health Insurance Portability and Accountability Act. All federal and state laws were followed. Informed consent was obtained from all survey participants via email. This study included academic ophthalmology departments with residency programs and excluded departments with preexisting sexual harassment programs that were felt to deviate significantly from the study. This study also excluded departments unable to obtain IRB approval and/or administer all surveys. We allowed for one-time crossover between study groups prior to the administration of surveys, if necessary, to maximize participation.

The authors contacted the 36 largest United States academic ophthalmology departments (based on resident numbers), primarily communicating with residency program directors and/or department chairs. Although results are less generalizable to smaller departments based on this study design, larger departments would capture a higher sample size. Of these, three programs declined due to existing sexual harassment requirements interfering with the research protocol. Three additional programs declined due to inadequate support for this research. Another seven declined without explanation. Of the remaining 23 programs, 11 were randomly assigned to the control group and 12 to the professionalism mentor group. We used a stratified randomization approach to evenly 
distribute programs with female chairs and/or residency program directors as well as top 10 programs based on U.S. News and World Report rankings. ${ }^{7}$ Subsequently, one program crossed over from control to professionalism mentor group due to a preexisting professionalism mentor-like role. Another program crossed over from professionalism mentor to control group due to onerous local IRB requirements for participating in the professionalism mentor group. There were seven programs that dropped out after randomization: two from each group due to failure to obtain IRB approval, one from the professionalism mentor group, and two from the control group due to lack of survey participation. Of the remaining 16 departments, nine were in the professionalism mentor group and seven were in the control group. Among the final groups, $3 / 9(33 \%)$ in the professionalism mentor group and $4 / 7$ (57\%) in the control group had a female chair and/or residency program director.

\section{Professionalism Mentor Group}

We specified that the professionalism mentor be an individual whom trainees and faculty would likely feel comfortable approaching; department chairs or program directors were excluded from this role. The residency program director and/or department chair typically selected the mentor from a voluntary faculty pool. Each professionalism mentor was advised to receive university bystander and victim advocate trainingusually through their Title IX office-primarily to learn their university's specific policies, resources, and procedures. The professionalism mentor's responsibilities included participating in a pre- and postsurvey, administering a 30- to 60-minute departmental educational session and introducing the new professionalism mentor role. Suggested educational curriculum included approaches to patient-initiated sexual harassment, bystander training, and confidentiality. Specific curriculum content was not distributed, however. Each university's Title IX department provided additional campus resources to most sessions. Although lack of uniformity disadvantaged the study, the authors felt that university-specific training and guidance were important for the relevance and utility of this program. For the next 6 to 10 months, the professionalism mentors served as a resource for dialog around and assistance with reporting complaints of sexual harassment. As a familiar faculty member, the professionalism mentor could provide trauma-informed support to individuals experiencing sexual harassment. Nonetheless, the professionalism mentor was asked to serve as a conduit to the Title IX office based on local university policy and not charged with investigation of any reported sexual harassment. Subsequent follow-up for the victim as well as remediation or other actions against the perpetrator were left to the discretion of the Title IX officers according to standard university, local, and federal policies and procedures. We selected the general title of professionalism mentor because it is more discreet than a title containing the words sexual harassment and allows for future expansion of the role to include other professionalism topics, such as racial discrimination. Other areas of professionalism outside of sexual harassment were not evaluated in this study, however.

\section{Control Group}

The control group was not assigned a professionalism mentor during the study period nor was an educational session organized through the research study. To maintain adequate enrollment, the study did not prohibit educational sessions or online educational modules if they were required by university policy or occurred as part of standard departmental curriculum over the course of the study period.

\section{Anonymous Survey}

The recently published ophthalmology sexual harassment survey ${ }^{1}$ informed development of the current survey, and an additional category of sex discrimination was included as recommended by the National Academies of Sciences, Engineering, and Medicine. ${ }^{4}$ Questions regarding feedback on the professionalism mentor, educational sessions, and departmental support were included. Each institution's representative (typically the professionalism mentor or residency program director) emailed the department's faculty and trainees the anonymous WebQ (Catalyst, Seattle, WA) survey before and after the 6- to 10-month study period, using a standardized email invitation containing embedded informed consent (-Supplementary Material S1, online only). The email notified prospective participants that clicking on the survey link constituted consent to participate with an optional $\$ 150$ incentive gift card raffle. The representative was encouraged to send reminder emails prior to the 1-week deadline. Each participating residency program director and professionalism mentor responded to a different online pre- and postsurvey including their opinion on the program, their assessment of their home ophthalmology department's response to unprofessional behavior, and formal complaints (requiring prior communication with their Title IX office). Some institutional IRB or Title IX policies prevented the residency program director from sharing this information, and instead the Title IX representative reached out to the first author (M.T.C.) directly. We did not exclude other institutions that prohibited any sharing of reporting data.

\section{Statistical Analysis}

The change in rate from pre- to postsurvey of reporting sexual harassment to an authority among faculty and trainees were compared between the two groups as the primary outcome measure. We also compared change in rate of anonymously reported sexual harassment. Two slightly differently worded Likert-style survey questions were included for professionalism mentor and educational session opinions. In general, a Cronbach's $\alpha$ reliability coefficient of 0.7 or higher is considered acceptable. ${ }^{8}$ A Cronbach's $\alpha$ ( 0.79 and 0.92 , respectively) confirmed internal consistency between the two questions, and the average was used for final analysis. The Cronbach's $\alpha$ statistic also validated identical questions for residency program directors ( 0.91 and 0.86 , respectively) and professionalism mentors ( 0.84 and 0.87 , respectively). Multinomial logistic regression was used for comparisons between groups in change from pre- to postsurvey. For all other analyses, Chisquare test assessed categorical data, and a $t$-test and ANOVA assessed continuous data. All analyses were based on the 
groups that participants ended in after crossover rather than intent-to-treat analysis. A two-sided $p$-value of $\leq 0.05$ was considered statistically significant. All analyses used SPSS V24 (IBM Corporation, New York City, NY).

\section{Results}

Among 16 participating ophthalmology departments, 605 faculty and trainees responded (adding both the pre- and postsurveys), with an unknown number of participants taking both the pre- and postsurveys. Of these, 16/605 (3\%) were prohibited by their IRB to reveal personal sexual harassment experiences, and 43/605 (7\%) failed to answer without explanation. Among the remaining 546 respondents, 86/546 (16\%) anonymously reported experiencing sexual harassment over the prior 10 months an average of $7.7 \pm 11.3$ times from $4.8 \pm 7.4$ perpetrators. Women represented $65 / 86$ (76\%) of sexual harassment victims. - Table 1 depicts the most common experiences, including offensive comments (28\%), unwanted attention (17\%), unwelcome verbal advances (13\%), and gender discrimination (17\%). Victims included residents (39\%) and academic attendings (49\%), while perpetrators were often patients and their family members $(44 \%)$ or academic attendings (35\%; - Table 1).

For the presurvey, there were 338 faculty/trainee participants with mean age $42.9( \pm 13.0$, range $=27-84)$. Of these, 40 (12\%) did not answer demographic questions. Of the remaining 298, 221 (74\%) were among nine departments randomized to the professionalism mentor group and 77 (26\%) were among the seven departments randomized to the control group. For the postsurvey, there were 267 faculty/trainee participants with a mean age of $43.9( \pm 12.8$, range $=27-84)$ years. Of these, $10(4 \%)$ did not answer demographic questions. Among the remaining 257 participants, 171 (67\%) were in the professionalism mentor group and 86/257 (34\%) were in the control group. Characteristics of the two groups are shown in - Table 2.

Geographic distribution of the two groups differed $(p<0.001$; postsurvey) but did not change from baseline (-Table 2). Demographics were similar between the two groups without change from baseline (-Table 2 ). Both the professionalism mentor and control groups did not experience a change in anonymously reported sexual harassment experiences over time $(p=0.85$ and $p=0.96$, respectively; $p=0.95$ comparing delta between groups, - Table 2). At baseline, the professionalism mentor group already experienced a lower rate of anonymously reported sexual harassment, although not statistically significant $(27 / 213$ [13\%] vs. $15 / 77$ [19\%]; presurvey, $p=0.15$ ). This difference was similar in the postsurvey $(22 / 163$ [13\%] vs. $17 / 86$ [20\%], $p=0.20)$. Number of experiences was similar between the two groups $(8.9 \pm 12.9$ vs. $7.5 \pm 10.7$, respectively; $p=0.75$ ). Programs with a professionalism mentor had an increase in rate of reporting to an authority from baseline (7-23\%), whereas the control group had a decrease from baseline (27-12\%). The difference in change over time between groups approached statistical significance ( $p=0.07$; - Table 2 ). Among those with access to a professionalism mentor, only $1 / 5$ (20\%) victims reporting
Table 1 Characteristics of sexual harassment experiences over prior 10 months among faculty and trainees responding to anonymous survey (all pre- and postsurveys included).

\begin{tabular}{|c|c|}
\hline $\begin{array}{l}\text { Specific sexual harassment } \\
\text { experiences, } n=86^{\mathrm{b}}\end{array}$ & $N(\%)^{a}$ \\
\hline Offensive comments & $52(28)$ \\
\hline Unwanted attention & $31(17)$ \\
\hline Unwelcome verbal advances & $25(13)$ \\
\hline Unwanted and persistent invitations & $7(4)$ \\
\hline Unwelcome explicit proposition & $2(1)$ \\
\hline Offensive displays & $5(3)$ \\
\hline Offensive body language & $18(10)$ \\
\hline Unwanted physical advances & $8(4)$ \\
\hline Sex discrimination & $32(17)$ \\
\hline Other & $7(4)$ \\
\hline \multicolumn{2}{|l|}{ Victim's gender, $n=86^{\mathrm{b}}$} \\
\hline Male & $17(20)$ \\
\hline Female & $65(76)$ \\
\hline Nonbinary/third gender & $1(1)$ \\
\hline Prefer not to say & $3(4)$ \\
\hline \multicolumn{2}{|l|}{ Victim's role, $n=86^{\mathrm{b}}$} \\
\hline Medical student ${ }^{c}$ & $1(1)$ \\
\hline Resident & 33 (39) \\
\hline Fellow & $6(7)$ \\
\hline Academic attending & $42(49)$ \\
\hline Other & $3(4)$ \\
\hline \multicolumn{2}{|l|}{ Perpetrator's role } \\
\hline Resident & $2(2)$ \\
\hline Fellow & $4(3)$ \\
\hline Academic attending & $45(35)$ \\
\hline Patient or patient's family member & $56(44)$ \\
\hline Residency program director & $1(1)$ \\
\hline Fellowship program director & $1(1)$ \\
\hline Department chair & $5(4)$ \\
\hline Faculty research mentor & $1(1)$ \\
\hline Other & $12(9)$ \\
\hline
\end{tabular}

aPercentage represents proportion of those who selected that answer among all responses. Allows for more than one response per participant; therefore, sum of values exceeds total " $n$ " and sum of percentages exceeds 100 .

${ }^{\mathrm{b}}$ Respondents experienced sexual harassment over the prior 10 months in 86 of 546 survey responses (16\%).

${ }^{\mathrm{C}} \mathrm{A}$ first-year resident responding to the survey described their experience when they were a medical student during the prior 10 months. Medical students were not surveyed in this study.

to an authority utilized the professionalism mentor. The remaining reporters in both groups reported to one or more of the following (percentages exceed 100 since individuals could select more than one): another academic attending within the department $(43 \%, 3 / 7)$, the residency program 
Table 2 Comparison of faculty and trainee opinions and experiences regarding sexual harassment over prior 10 months for programs randomized to control group versus professionalism mentor group based on anonymous pre- and postsurvey

\begin{tabular}{|c|c|c|c|c|c|c|c|}
\hline & \multicolumn{3}{|c|}{$\begin{array}{l}\text { Professionalism mentor } \\
N(\%)\end{array}$} & \multicolumn{3}{|l|}{$\begin{array}{l}\text { Control } \\
N(\%)\end{array}$} & \multirow[t]{2}{*}{$p$-Value } \\
\hline & Pre & Post & $p$-Value ${ }^{\mathrm{b}}$ & Pre & Post & $p$-Value ${ }^{\text {b }}$ & \\
\hline & $n=221^{c}$ & $n=171^{c}$ & & $n=77^{c}$ & $n=86^{c}$ & & \\
\hline \multicolumn{8}{|l|}{ Gender } \\
\hline Male & $105(48)$ & $92(54)$ & \multirow[t]{3}{*}{0.62} & $45(58)$ & $49(57)$ & \multirow[t]{3}{*}{0.97} & \multirow[t]{3}{*}{0.60} \\
\hline Female & 109 (49) & $75(44)$ & & $30(39)$ & $35(41)$ & & \\
\hline Other & $7(3)$ & $4(2)$ & & $2(3)$ & $2(2)$ & & \\
\hline \multicolumn{8}{|l|}{ Race } \\
\hline White & $159(69)$ & $115(64)$ & \multirow[t]{5}{*}{0.47} & $52(67)$ & $56(64)$ & \multirow[t]{5}{*}{0.96} & \multirow[t]{5}{*}{0.18} \\
\hline Black & $3(1)$ & $1(1)$ & & $1(1)$ & $3(3)$ & & \\
\hline Asian & $56(24)$ & $46(26)$ & & $15(19)$ & $16(18)$ & & \\
\hline Hispanic & $3(1)$ & $2(1)$ & & $3(4)$ & $3(3)$ & & \\
\hline Other & $9(4)$ & $15(8)$ & & $7(9)$ & $9(10)$ & & \\
\hline \multicolumn{8}{|l|}{ Geographic region } \\
\hline South & $0(0)$ & $0(0)$ & \multirow[t]{4}{*}{0.40} & $27(35)$ & $22(26)$ & \multirow[t]{4}{*}{0.48} & \multirow[t]{4}{*}{0.70} \\
\hline Northeast & $38(17)$ & $23(13)$ & & $18(23)$ & $26(30)$ & & \\
\hline West & $114(52)$ & $85(50)$ & & $9(12)$ & $8(9)$ & & \\
\hline Midwest & $69(31)$ & $63(37)$ & & $23(30)$ & $30(35)$ & & \\
\hline Department supports reporting & $184(83)$ & $150(88)$ & 0.46 & $64(83)$ & $69(80)$ & 0.50 & 0.72 \\
\hline Professionalism mentor would be useful $^{\mathrm{d}}$ & $138(62)$ & $116(68)$ & 0.15 & $43(56)$ & $52(60)$ & 0.41 & 0.75 \\
\hline Educational sessions would be usefuld $^{d}$ & $126(57)$ & $110(64)$ & 0.006 & $35(45)$ & $58(67)$ & 0.02 & 0.53 \\
\hline \multicolumn{8}{|l|}{ Departments should have: } \\
\hline Professionalism mentor + educational session & $146(66)$ & $116(68)$ & \multirow[t]{3}{*}{0.93} & $46(60)$ & $51(59)$ & \multirow[t]{3}{*}{0.12} & \multirow[t]{3}{*}{0.57} \\
\hline Educational session alone & $55(25)$ & $40(23)$ & & $21(27)$ & $31(36)$ & & \\
\hline Neither & $20(9)$ & $15(9)$ & & $10(13)$ & $4(5)$ & & \\
\hline Respondents $^{\mathrm{e}}$ & $n=213$ & $n=163$ & & $n=77$ & $n=86$ & & \\
\hline Experienced sexual harassment & $27(13)$ & $22(13)$ & 0.85 & $15(19)$ & $17(20)$ & 0.96 & 0.95 \\
\hline Reported sexual harassment $^{f}$ & $2(7)$ & $5(23)$ & 0.22 & $4(27)$ & $2(12)$ & 0.37 & 0.07 \\
\hline
\end{tabular}

Abbreviation: N/A, not applicable.

aDifference between pre- and postsurvey were compared between professionalism mentor and control groups by using multinomial logistic regression.

${ }^{b}$ Chi-square test was used for all comparisons of categorical data, and $t$ test and ANOVA for continuous data. For Likert's questions, $p$-values compare overall results, even though only strongly agree or agree are shown.

'Excludes 50 respondents who did not answer demographic questions. For this reason, these numbers differ from that of $\boldsymbol{\sim}$ Table 1 .

${ }^{\mathrm{d}}$ Averages the results of two differently worded Likert style survey questions.

eExcludes 16 additional respondents who did not answer the sexual harassment question.

${ }^{\mathrm{f}}$ Among those who experienced sexual harassment.

director $(57 \%, 4 / 7)$, the university ombudsperson office $(14 \%$, $1 / 7)$, department chair $(43 \%, 3 / 7)$, the university advocacy office $(14 \%, 1 / 7)$, or other $(43 \%, 3 / 7)$. Location in the Northeast $(6 \%, 6 / 105)$ or West $(13 \%, 28 / 216)$ was associated with lower anonymously reported sexual harassment compared with the South $(16 \%, 8 / 49)$ or Midwest $(21 \%, 39 / 185 ; p<0.001)$. Departments led by a female chair and/or program director had similar anonymously reported sexual harassment compared with all-male-led departments (24/187 [13\%] vs. 57/352 [16\%], respectively, with 16 unable to respond, $p=0.31$ ).
When asked whether the department supports faculty and trainees who report unprofessional behavior, $88 \%$ in the professionalism mentor group and $80 \%$ in the control group responded "agree" or "strongly agree" ( $p=0.15$, postsurvey; $p=0.72$ comparing change from baseline; - Table 2). The professionalism mentor group favored the concept of a professionalism mentor at a similar rate to the control group (68 vs. $60 \%$, postsurvey; $p=0.49$ ). These opinions did not significantly change over time for either group $(p=0.75$ comparing change from baseline between groups). In 
contrast, both groups developed a more favorable opinion toward the educational sessions from the pre- to postsurvey ( $p=0.006$ and $p=0.02$, respectively; $p=0.53$ comparing change between groups). Both professionalism mentor and control groups recommended both professionalism mentor and educational session for academic departments (68 vs. $59 \%$, respectively; postsurvey) as opposed to educational session alone ( 23 vs. $36 \%$, respectively; postsurvey). These trends did not change over time ( $p=0.57$ comparing change from baseline between groups).

All nine professionalism mentors responded to both preand postsurveys (100\%). All 6/7 (86\%) residency program directors in the control group responded to both surveys. All nine $(100 \%)$ residency program directors in the professionalism mentor group responded to the presurvey, while 8/9 (89\%) responded to the postsurvey. Among the remaining participants, all (100\%) residency program directors in both groups agreed with the statement that their department supports trainees and faculty who report unprofessional behavior in both surveys. Residency program directors and professionalism mentors in the professionalism mentor group strongly believed in the efficacy of both a professionalism mentor and educational program in both surveys. The program directors in the control group had less enthusiastic opinions regarding both these approaches ( $p=0.03$; postsurvey), though neither group significantly changed from baseline ( $p=0.67$ comparing change between groups; - Table 3). For the presurvey, all nine (100\%) residency program directors from the professionalism mentor group, and all six (100\%) from the control group identified no departmental complaints received by their Title IX office over the prior 10 months (one control Title IX department notified the lead author directly). For the postsurvey, 2/8 (25\%) from the professionalism mentor group and $1 / 6(17 \%)$ from the control group were prohibited from answering this question. Among 11 remaining departments, only one (9\%), from the control group launched a Title IX complaint.

\section{Discussion}

In a recent survey of 7,409 surgical residents (comprising 99.3\% of the participants in the 2018 American Board of Surgery InTraining Examination), 31.9\% reported gender discrimination and $10.3 \%$ reported frank sexual harassment. ${ }^{9}$ Rates of gender discrimination and sexual harassment were higher among women, with 65.1 and $19.9 \%$ of women reporting episodes of these mistreatments, respectively, associated with higher risk of symptoms of burnout (odds ratio: 2.94; 95\% confidence interval [CI]: 2.58-3.36) and suicidal thoughts (odds ratio: 3.07; 95\% CI: $2.25-4.19) .{ }^{9}$ In a recent survey of ophthalmologists, $59 \%$

Table 3 Comparison of residency program director and professionalism mentor opinions regarding sexual harassment over prior 10 months for programs randomized to control group versus professionalism mentor group based on anonymous pre- and postsurvey

\begin{tabular}{|c|c|c|c|c|c|c|c|}
\hline \multirow[t]{2}{*}{ Respondents } & \multicolumn{3}{|c|}{$\begin{array}{l}\text { Professionalism Mentor } \\
N(\%)\end{array}$} & \multicolumn{3}{|l|}{$\begin{array}{l}\text { Control } \\
N(\%)\end{array}$} & \multirow[t]{2}{*}{$p$-Value ${ }^{a}$} \\
\hline & Pre & Post & $p$-Value ${ }^{b}$ & Pre & Post & $p$-Value ${ }^{b}$ & \\
\hline Residency program directors & $n=9$ & $n=8$ & & $n=6$ & $n=6$ & & \\
\hline Department supports reporting & $9(100)$ & $8(100)$ & 1.00 & $6(100)$ & $6(100)$ & 1.00 & 1.00 \\
\hline Professionalism Mentor would be useful ${ }^{\mathrm{c}}$ & $8(89)$ & $8(100)$ & 0.52 & $3(50)$ & $3(50)$ & 1.00 & 0.53 \\
\hline Educational sessions would be useful ${ }^{\mathrm{c}}$ & $8(89)$ & $8(100)$ & 0.52 & $4(67)$ & $5(83)$ & 0.66 & 0.41 \\
\hline \multicolumn{8}{|l|}{ Academic medical departments should have: } \\
\hline Professionalism mentor + educational session & $9(100)$ & $8(100)$ & \multirow[t]{3}{*}{1.00} & $4(67)$ & $3(50)$ & \multirow[t]{3}{*}{0.58} & \multirow[t]{3}{*}{0.67} \\
\hline Educational session alone & $0(0)$ & $0(0)$ & & $2(33)$ & $3(50)$ & & \\
\hline Neither & $0(0)$ & $0(0)$ & & $0(0)$ & $0(0)$ & & \\
\hline Professionalism mentors & $n=9$ & $n=9$ & & \multicolumn{3}{|l|}{$\mathrm{N} / \mathrm{A}$} & $\mathrm{N} / \mathrm{A}$ \\
\hline Department supports reporting & $7(78)$ & $8(89)$ & 0.52 & \multicolumn{3}{|l|}{$\mathrm{N} / \mathrm{A}$} & $\mathrm{N} / \mathrm{A}$ \\
\hline Professionalism Mentor would be useful $^{b}$ & $8(89)$ & $8(89)$ & 0.15 & \multicolumn{3}{|l|}{ N/A } & $\mathrm{N} / \mathrm{A}$ \\
\hline 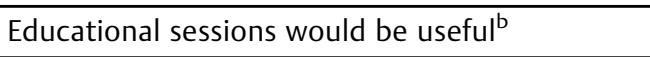 & $8(89)$ & $8(89)$ & 0.58 & \multicolumn{3}{|l|}{$\mathrm{N} / \mathrm{A}$} & $\mathrm{N} / \mathrm{A}$ \\
\hline \multicolumn{8}{|l|}{ Academic medical departments should have: } \\
\hline Professionalism mentor + educational session & $8(89)$ & $7(78)$ & \multirow[t]{3}{*}{1.00} & \multirow{3}{*}{\multicolumn{3}{|c|}{$\mathrm{N} / \mathrm{A}$}} & \multirow[t]{3}{*}{$\mathrm{N} / \mathrm{A}$} \\
\hline Educational session alone & $1(11)$ & $2(22)$ & & & & & \\
\hline Neither & $0(0)$ & $0(0)$ & & & & & \\
\hline
\end{tabular}

Abbreviation: N/A, not applicable.

${ }^{a}$ Difference between pre- and postsurvey were compared between professionalism mentor and control groups using multinomial logistic regression. ${ }^{b}$ Chi-square test was used for all comparisons of categorical data, and $t$ test and ANOVA for continuous data. For Likert questions, $p$-values compare overall results, even though only strongly agree or agree are shown.

${ }^{c}$ Averages the results of two differently worded Likert-style survey questions. 
reported experiencing sexual harassment, ${ }^{1}$ with $25 \%$ impacting their ability to work and $15 \%$ resulting in changed jobs or careers. The reporting rate was only $15 \%$. We set out to determine whether a professionalism mentor could help improve workplace climate and sexual harassment reporting within academic ophthalmology departments.

In this study, anonymously reported rates of sexual harassment over two 10-month periods were high (16\%) among 546 male and female faculty and trainee respondents across 16 academic institutions. As in prior studies, ${ }^{9}$ most victims were females (76\%), including both faculty $(49 \%)$ and residents (39\%). Programs with professionalism mentors had an increase in incidents reported to an authority from baseline (7-23\%) compared with the control group, which had a decrease from baseline (27-12\%). The reasons for a decreasing reporting rate in the control group are unknown, but possible explanations include decreasing enthusiasm for the \#MeToo movement over time and/or perceived increasing backlash against the movement. ${ }^{10}$ The difference in change from baseline between groups approached statistical significance in this brief study ( $p=0.07$ ).

The National Academies of Sciences, Engineering, and Medicine recently published a comprehensive review of sexual harassment literature in academic departments revealing that departmental and institutional culture, including leadership attitudes and subtle forms of harassment (i.e., microaggressions), are the strongest predictors of sexual harassment rate and severity. ${ }^{4}$ The professionalism mentor is one approach to addressing departmental culture. Obstacles to successfully implementing a professionalism mentor program include a lack of leadership commitment and inadequate funding or interest in the voluntary role. Nonetheless, faculty seemed eager to take this on. As one professionalism mentor commented: "[The professionalism mentor] is an opportunity to have a key person in the department serve as the go-to person for reporting, organizing educational events, and being the grassroots cheerleader for spreading awareness and understanding of sexual harassment. When you just bring in outsiders to educate or receive reporting, there is skepticism and distrust." Another professionalism mentor focused on the issue of gender and leadership: "I think particularly in a department like mine, with men in all positions of leadership, having a female professionalism mentor is absolutely critical in the residents feeling supported in reporting any incidences of harassment." For these reasons and others, most faculty and trainees in the interventional arm of this study recommended instituting a professionalism mentor with educational session (68\%), compared with educational session alone $(23 \%)$ or neither (9\%). Residency program directors in the interventional arm were even more enthusiastic, with $100 \%$ recommending a professionalism mentor with educational session program. Nonetheless, the sexual harassment rate did not change from the pre- to the postsurvey, regardless of randomization. These results seem promising, but a large shift in culture may take more time than was assessed in this study.

Only one report was provided to the professionalism mentor during this 10-month period, suggesting that the reporting role of the professionalism mentor may have been less impactful than the educational role. Sexual harassment education is a necessary component of any prevention program, ${ }^{6}$ both to encourage reporting of serious unprofessional behavior and to learn to respond to milder forms of sexual harassment directly as a victim or bystander. Bystander training is one of the few interventions shown to successfully prevent sexual harassment. ${ }^{11}$ Training can help prepare clinicians to provide direct feedback to patients who initiate sexual harassment, although education on reporting mechanisms for rare serious allegations is also necessary. ${ }^{12}$ These steps help build a departmental culture and climate in which individuals feel empowered to speak out against and ultimately prevent sexual harassment.

The American Academy of Ophthalmology endorsed a clear zero tolerance policy toward sexual harassment in $2018 .^{13}$ Rule 18 was later added to the code of ethics, creating an enforceable antiharassment and discrimination standard for all fellows and members. ${ }^{14}$ Despite existing structures and policies, sexual harassment and underreporting continue in academics. The Academy and individual departments must explore new ways to address this problem. While anonymous mechanisms and nondepartmental ombudsman are crucial, a departmental professionalism mentor can clarify institutional reporting mechanisms to minimize barriers to reporting more serious forms of harassment while creating educational opportunities to learn preventative strategies. Clear definitions and processes reviewed on a semiannual basis by a designated individual may help reduce confusion among residents and faculty faced with deciding what, when, and how to report incidents of harassment. Nonetheless, this study did not establish the feasibility of a professionalism mentor outside a university setting, where lack of an investigative Title IX infrastructure may introduce greater complexity to the role.

The results of our study should be understood within the context of its limitations. Importantly, this study was based on a voluntary survey from a select number of ophthalmology departments in which there may be bias toward overreporting (victims may be more likely to participate) or under-reporting (victims may be frightened to participate). The higher response rate, greater program director enthusiasm, and lack of Southern states (identified to have a higher sexual harassment rate) in the professionalism mentor group may also bias the study results. Furthermore, we had seven programs drop out and two groups switch after randomization, which may have led to unintended bias. Because the control group did not exclude programs who went on to require sexual harassment educational sessions or modules, this study may have underestimated the impact of the professionalism mentor. Moreover, professionalism mentors were selected by departmental leadership; it is possible that a different selection process would have resulted in a more effective individual. Finally, given the large number of departments included with individual departmental listservs, it is not possible to determine how many surveys were sent and received, whether more than one response came from an individual and a response rate cannot be obtained. 
Despite its limitations, this study is the first to examine a method by which to improve the reporting of sexual harassment in academic ophthalmology departments. We are hopeful that departments across the country will consider a more formalized approach to education and support of trainees and faculty with regard to this important issue.

\section{Funding}

This work was supported by unrestricted grants from Research to Prevent Blindness and the NIH CORE Grant (grant number EY001730) to the University of Washington Department of Ophthalmology and an unrestricted grant from Research to Prevent Blindness to the University of California, Los Angeles Department of Ophthalmology. The sponsors had no participation in the study design, collection, analysis, and interpretation of data; writing of the report, and the decision to submit the article for publication.

\section{Conflict of Interest}

None declared.

\section{Acknowledgments}

The authors are grateful to Lynn Gordon, MD, PhD, Stein Eye Institute, Department of Ophthalmology, UCLA, Los Angeles, CA, for critical review of this manuscript. They are also thankful to all participating institutions in this multicenter study. Participating institutions requested that we not reveal their identity due to the sensitive nature of the study results.

\section{References}

1 Cabrera MT, Enyedi LB, Ding L, MacDonald SM. Sexual harassment in ophthalmology: a survey study. Ophthalmology 2019;126(01): $172-174$
2 Jagsi R, Griffith KA, Jones R, Perumalswami CR, Ubel P, Stewart A. Sexual harassment and discrimination experiences of academic medical faculty. JAMA 2016;315(19):2120-2121

3 Fnais N, Soobiah C, Chen MH, et al. Harassment and discrimination in medical training: a systematic review and meta-analysis. Acad Med 2014;89(05):817-827

4 National Academies of Sciences, Engineering, and Medicine. Sexual Harassment of Women: Climate, Culture, and Consequences in Academic Sciences, Engineering, and Medicine. Washington, DC: The National Academies Press; 2018

5 Carr PL, Ash AS, Friedman RH, et al. Faculty perceptions of gender discrimination and sexual harassment in academic medicine. Ann Intern Med 2000;132(11):889-896

6 Freedman-Weiss MR, Chiu AS, Heller DR, et al. Understanding the barriers to reporting sexual harassment in surgical training. Ann Surg 2020;271(04):608-613

7 Best Hospitals for Ophthalmology. US News and World Report 2017. Accessed August 2, 2018 at: https://health.usnews.com/best-hospitals/rankings/ophthalmology

8 Cortina JM. What is coefficient alpha? An examination of theory and applications. J Appl Psychol 1993;78:98-104

9 Hu YY, Ellis RJ, Hewitt DB, et al. Discrimination, abuse, harassment, and burnout in surgical residency training. N Engl J Med 2019;381(18):1741-1752

10 Soklaridis S, Zahn C, Kuper A, Gillis D, Taylor VH, Whitehead C. Men's fear of mentoring in the \#metoo era - what's at stake for academic medicine? N Engl J Med 2018;379(23):2270-2274

11 Potter SJ, Stapleton JG. Translating sexual assault prevention from a college campus to a United States military installation: piloting the know-your-power bystander social marketing campaign. J Interpers Violence 2012;27(08):1593-1621

12 Warsame RM, Hayes SN. Mayo clinic's 5-step policy for responding to bias incidents. AMA J Ethics 2019;21(06):E521-E529

13 Parke DW. Sexual harassment and ophthalmology. EyeNet Magazine. 2018;22:12

14 Code of Ethics. American Academy of Ophthalmology 2020. Accessed September 8, 2020 at: https://www.aao.org/ethics-detail/code-of-ethics\#harass 